«Pollution, carrying capacity and the Allee effect »

\author{
$\underline{\text { Auteurs }}$
}

Stefano Bosi, David Desmarchelier

Document de Travail $\mathrm{n}^{\circ} 2018-37$

Septembre 2018

Bureau d'Économie

Théorique et Appliquée

BETA

www.beta-umr7522.fr

@beta_economics

Contact :

jaoulgrammare@beta-cnrs.unistra.fr 


\title{
Pollution, carrying capacity and the Allee effect
}

\author{
Stefano BOSI \\ EPEE, Université Paris-Saclay \\ David DESMARCHELIER \\ University of Lorraine, University of Strasbourg, AgroParisTech, CNRS, INRA, BETA
}

September 10, 2018

\begin{abstract}
In ecology, one of the simplest representation of population dynamics is the logistic equation. This basic view can be enriched by considering two important variables: (1) the maximal population density Nature can support (carrying capacity) and (2) the critical density threshold under which the population disappear (Allee effect). The economic literature on biodiversity and renewable resources ignores both these variables. Evidence suggests also that these variables are affected by the pollution level due to economic activity. Indeed, a degraded environment is unsuitable for wildlife and reduces the carrying capacity, while the climate change entails the habitat fragmentation and, lowering the wildlife reproduction possibilities, raises the Allee effect. The present paper aims to incorporate both endogenous carrying capacity and Allee effect in a Ramsey model augmented with biodiversity as a renewable resource. Our extended framework enables us to study the effect of a Pigouvian tax on anthropogenic mass extinction. We find that, when the household overvalues biodiversity with respect to consumption, a higher green-tax rate is beneficial in three respects entailing: (1) a lower pollution and a higher biodiversity, (2) a welfare improvement and (3) a less likely mass extinction.
\end{abstract}

Keywords: Allee effect, carrying capacity, pollution, Ramsey model, logistic dynamics, Hopf bifurcation.

JEL Classification: E32, O44.

\section{Introduction}

A number of economists have studied the dynamics of renewable resources and biodiversity in models of capital accumulation. To the best of our knowledge, the seminal paper on the interaction between artificial and natural capital accumulation in a Ramsey model is Beltratti et al. (1994). These authors consider the renewable resource as a productive input and a pollution externality coming from consumption and impairing the resource evolution. They show that 
the saddle-path stability is preserved under the assumption of a small resource impact on production. Ayong Le Kama (2001) revisits their work taking in account production instead of consumption as source of pollution. As in Beltratti et al. (1994), he recovers the saddle-path stability, but without the restrictive assumption of a small resource effect on production.

In Wirl (2004), the renewable resource is no longer productive but enters the utility function, which is separable in consumption and resource. Using a logistic function (à la Pearl-Verhulst) to represent the inverted-U-shaped reproduction function of Nature, he finds two steady states: the one along the upward-sloping branch of this function, the other along the downward-sloping branch. Wirl (2004) points out the occurrence of a limit cycle around the lower steady state through a Hopf bifurcation and the absence of complex dynamics around the higher one. More recently, Bosi and Desmarchelier (2018b) revisit Wirl (2004) in two respects considering: (1) a market economy instead of a central planner, (2) a non-separable utility function. They reach an opposite conclusion: only the higher steady state (along the decreasing branch of the reproduction function) can be surrounded by a limit cycle. They also find a saddle-node bifurcation if the environmental impact of production is sufficiently large. In this case, the two steady states collide and disappear. Eventually, they show that the joint occurrence of saddle-node and Hopf bifurcation gives rise to a parasitic loop when the two steady states coalesce through a Bogdanov-Takens bifurcation.

All these results rest on a simple representation of reproduction function: the logistic equation. Such unsophisticated mechanics misses two important features of population dynamics and biodiversity. On the one hand, Nature does not support an infinite number of individuals and faces a carrying capacity. $^{1}$ On the other hand, since the Allee (1931) pioneering work, biologists are aware that below a critical density level, many plants and animals experience an extinction vortex. This density threshold is precisely known as Allee effect. Following Courchamp et al. (1999) the prevailing interpretation of this effect is the shortage of receptive mate encounters during the mating period when the density is too low.

There is little doubt that pollution induced by economic activities affects both the carrying capacity and the Allee effect. Indeed, a degraded environment is recognized as a low biodiversity area. For example, according to Celli and Maccagnani (2003), pollutants as pesticides are responsible for bees extinction generating a stress for many plants and compromising in turn their survival (Memmott et al. (2004)). It is reasonable to expect that the carrying capacity is a decreasing function of pollution. In addition, pollution seems to influence the Allee effect. As reported by Brook et al. (2008) among others, pollution reduces the fertilization success. It is justified to define the Allee effect as an increasing function of pollution.

Taking into account both the carrying capacity and the Allee effect, and the joint effect of pollution becomes relevant to represent an anthropogenic mass extinction. Indeed, if the carrying capacity falls below the Allee threshold

\footnotetext{
${ }^{1}$ See Courchamp et al. (1999) among others.
} 
because of man-made pollution, then Nature will support a lower biodiversity level than what is needed to ensure biodiversity regeneration, and will enter an extinction vortex induced by polluting economic activities. There is a lot of evidences of such an anthropogenic mass extinction (see Barnosky et al. (2011) and Ceballos et al. (2015) among others).

Considering the joint effect of pollution on the carrying capacity and the Allee threshold is also relevant to understand the role of green policies in the anthropogenic mass extinction. For this purpose, we build a Ramsey model where a pollution externality, viewed as a stock coming from production, affects the evolution of a renewable resource (interpreted as biodiversity) through both the carrying capacity and the Allee effect. The government levies a simple Pigouvian tax on production activities to finance depollution. In the long run, the economy exhibits multiple regimes: a steady state with zero biodiversity corresponding to a mass extintion, and two steady states driven by the carrying capacity and the Allee effect respectively. The fiscal authority is concerned by the equilibrium transitions to these steady states. In the short run, we observe the possibility of both transcritical and Hopf bifurcations. The Hopf bifurcation can occur only near the steady state governed by the carrying capacity and entails the emergence of a limit cycle around. The transcritical bifurcation can occur when the inertia of pollution stock is sufficiently large. In this case, the Allee threshold meets the carrying capacity and the mass extinction takes place. Interestingly, when households overvalue the biodiversity with respect to consumption, a higher green-tax rate has a triple benefit: (1) a lower pollution jointly with a higher biodiversity, (2) a welfare improvement and (3) a mass extinction less likely.

The paper is organized as follows: section 2 presents the model, sections 3 , 4 and 5 study respectively the equilibrium, the steady state and the welfare. Dynamics are analyzed in section 6 and illustrated through a simulation in section 7 . Section 8 concludes the paper. All the proof are gathered in the Appendix.

\section{The model}

\section{$2.1 \quad$ Firms}

Each firm chooses the amount of capital and labor to maximize the profit taking as given both the real interest rate $r$ and the wage rate $w$. In addition, the government levies a proportional tax $\tau \in(0,1)$ on polluting production $F\left(K_{j}, L_{j}\right)$ of firm $j$ to finance the maintenance of natural resource.

Assumption 1 The production function $F: \mathbb{R}_{+}^{2} \rightarrow \mathbb{R}_{+}$is $C^{2}$, homogeneous of degree one, strictly increasing and concave. Inada conditions hold.

The profit maximization $\max _{K_{j}, L_{j}}\left[F\left(K_{j}, L_{j}\right)-r K_{j}-w L_{j}-\tau F\left(K_{j}, L_{j}\right)\right]$ entails the following first-order conditions:

$$
r=(1-\tau) f^{\prime}\left(k_{j}\right) \text { and } w=(1-\tau)\left[f\left(k_{j}\right)-k_{j} f^{\prime}\left(k_{j}\right)\right]
$$


where $k_{j} \equiv K_{j} / L_{j}$ is the capital intensity and $f\left(k_{j}\right) \equiv F\left(k_{j}, 1\right)$ the average productivity of the firm $j$.

All the firms share the same technology and address the same demand for capital.

Corollary 1 Let $k \equiv K / L$ with $K \equiv \sum_{j=1}^{J} K_{j}$ and $L \equiv \sum_{j=1}^{J} L_{j}$. In aggregate terms, $Y=F(K, L)$ and profit maximization yields

$$
r=(1-\tau) \rho(k) \text { and } w=(1-\tau) \omega(k)
$$

with $\rho(k) \equiv f^{\prime}(k)$ and $\omega(k) \equiv f(k)-k f^{\prime}(k)$.

We introduce the capital share in total disposable income and the elasticity of capital-labor substitution:

$$
\alpha(k) \equiv \frac{r k}{(1-\tau) f(k)}=\frac{k f^{\prime}(k)}{f(k)} \text { and } \sigma(k)=\alpha(k) \frac{\omega(k)}{k \omega^{\prime}(k)}
$$

In addition, we determine the elasticities of factor prices:

$$
\frac{k \rho^{\prime}(k)}{\rho(k)}=-\frac{1-\alpha(k)}{\sigma(k)} \text { and } \frac{k \omega^{\prime}(k)}{\omega(k)}=\frac{\alpha(k)}{\sigma(k)}
$$

\subsection{Households}

The representative household earns a capital income $r h$ where $h$ denotes the individual wealth at time $t$ and a labor income $w l$ with $l=1$ (inelastic labor supply). Thus, the household consumes and saves her income according to the budget constraint:

$$
c+\dot{h} \leq(r-\delta) h+w
$$

where $\dot{h}$ denotes the time-derivative of wealth. The gross investment includes the capital depreciation at the rate $\delta$.

Let $u(c, N)$ be the utility function of the representative household where $N$ represents an aggregate renewable resource which can be assimilated to the biodiversity level. We assume that biodiversity affects marginal utility of consumption $\left(u_{c N} \neq 0\right)$. If biodiversity increases the consumption demand, biodiversity and consumption are complement $\left(u_{c N}>0\right)$ : it is the case when households like to consume in a pleasant environment, in presence of a large biodiversity. Conversely, if biodiversity lowers consumption demand, then biodiversity and consumption are substitutable: in this case, the household compensates the utility loss due to a loss of biodiversity by increasing her consumption demand $\left(u_{c N}<0\right)$. For now, we do not impose any restriction.

Assumption 2 Preferences are rationalized by a non-separable utility function $u(c, N)$. First and second-order restrictions hold on the sign of derivatives: $u_{c}>0, u_{N}>0$ and $u_{c c}<0$, jointly with the limit conditions: $\lim _{c \rightarrow 0^{+}} u_{c}=\infty$ and $\lim _{c \rightarrow+\infty} u_{c}=0$. 
We introduce the second-order elasticities:

$$
\left[\begin{array}{cc}
\varepsilon_{c c} & \varepsilon_{c N} \\
\varepsilon_{N c} & \varepsilon_{N N}
\end{array}\right] \equiv\left[\begin{array}{cc}
\frac{c u_{c c}}{u_{c}} & \frac{N u_{c N}}{u_{c}} \\
\frac{c u_{N c}}{u_{N}} & \frac{N u_{N N}}{u_{N}}
\end{array}\right]
$$

$-1 / \varepsilon_{c c}$ represents the intertemporal elasticity of substitution in consumption while $\varepsilon_{c N}$ captures the effect of the natural resource on the marginal utility of consumption. Typically, if $\varepsilon_{c N}>0(<0)$, then the natural resource and consumption are complement (substitute) for households.

In a Ramsey model, the representative household maximizes an intertemporal utility functional:

$$
\int_{0}^{\infty} e^{-\theta t} u(c, N) d t
$$

under the budget constraint (2) where $\theta>0$ denotes the rate of time preference.

Proposition 2 The first-order conditions of the consumer's program are given by a static relation

$$
\mu=u_{c}(c, N)
$$

a dynamic Euler equation

$$
\dot{\mu}=\mu(\theta+\delta-r)
$$

and the budget constraint (2), now binding,

$$
\dot{h}=(r-\delta) h+w-c
$$

jointly with the transversality condition $\lim _{t \rightarrow \infty} e^{-\theta t} \mu(t) h(t)=0 . \quad \mu$ denotes the multiplier associated to the budget constraint.

Applying the Implicit Function Theorem to the static relation (4), we obtain the consumption function $c \equiv c(\mu, N)$ with elasticities

$$
\frac{\mu}{c} \frac{\partial c}{\partial \mu}=\frac{1}{\varepsilon_{c c}}<0 \text { and } \frac{N}{c} \frac{\partial c}{\partial N}=-\frac{\varepsilon_{c N}}{\varepsilon_{c c}}
$$

\subsection{Biodiversity}

Biodiversity behaves as a biological population. According to Courchamp et al. (1999) among others, population dynamics can be described by the following single equation:

$$
\dot{N}=N\left(\frac{N}{A}-1\right)\left(1-\frac{N}{C}\right)
$$

where $N$ is the population while $A$ and $C$ represent respectively the Allee effect (the positive correlation between population density and individual fitness) and the carrying capacity (the number of people, animals, or crops a region can support without environmental degradation). In his seminal work, Allee (1931) observed a decrease in the growth rate of many animals and plant when they reach a sufficiently low density. According to Courchamp et al. (1999), the 
dominant interpretation of this effect has been introduced by Allee himself: the shortage of receptive mate encounters during the mating period when density is too low. For instance, Brook et al. (2008) show that environmental pollutants reduce fertilization success and exacerbate the Allee effect. Formally, $A$ is an increasing function of the pollution level $P$. Conversely, habitat degradation implies a lower carrying capacity. Formally, $C$ is a decreasing function of pollution. The following assumption sums up the pollution effects on the Allee effect and the carrying capacity. ${ }^{2}$

Assumption $3 A \equiv A(P)$ and $C \equiv C(P)$ with $A^{\prime}(P) \geq 0$ and $C^{\prime}(P) \leq 0$.

We introduce the first-order elasticities of the Allee effect and the carrying capacity:

$$
\varepsilon_{A} \equiv \frac{P A^{\prime}(P)}{A(P)} \geq 0 \text { and } \varepsilon_{C} \equiv \frac{P C^{\prime}(P)}{C(P)} \leq 0
$$

Example In the bifurcation analysis, we will consider locally isoelastic functions (around the steady state):

$$
A(P) \equiv \bar{A} P^{\varepsilon_{A}} \text { and } C(P) \equiv \bar{C} P^{\varepsilon_{C}}
$$

$A$ and $C$ are constant if $\varepsilon_{A}=\varepsilon_{C}=0$.

We remark that equation (8) implies three steady states: $N_{0}=0, N_{1}=A$ and $N_{2}=C$.

If $A$ and $C$ are constant and $A<C$, then $N_{0}$ and $N_{2}$ are stable while $N_{1}$ is unstable. This means that, if $N_{0}<N<N_{1}$, a mass extinction is underway (Allee effect). However, if $N>N_{1}$, wildlife is preserved and the system converges to the highest steady state $N_{2}$. If $A$ and $C$ are constant and $A>C, N_{0}$ and $N_{1}$ are stable while $N_{2}$ is unstable.

If $A$ and $C$ are no longer constant and depend on $P$ (Assumption 3), dynamics become more complex. In particular, the pollution channel can promote the occurrence of persistent cycles as we will see in section 6 .

\subsection{Government}

The government spends all the tax revenues to finance depollution expenditures $G$ according to the following balanced budget rule:

$$
G=\tau F(K, L)
$$

\subsection{Pollution}

The aggregate stock of pollution $P$ is a pure externality coming from production. The government takes care of depollution through the abatement expenditures $G$. The pollution accumulation follows a linear process:

$$
\dot{P}=-a P+b Y-m G
$$

\footnotetext{
${ }^{2}$ Models with a renewable resource and pollution often consider the following logistic law augmented by a linear effect of pollution: $\dot{N}=N(1-N)-\xi P$ with $0 \leq \xi \leq 1$ (see Wirl (2004) among others). Equation (8) differs because the pollution impact passes through the carrying capacity and the Allee effect, that, thus, become endogenous variables.
} 
$a \geq 0, b \geq 0$ and $m \geq 0$ capture respectively the natural rate of pollution absorption, the environmental impact of production and the pollution abatement efficiency. We normalize the population to the unity: $L=1$, and the process (11) becomes in intensive terms: $\dot{P}=-a P+(b-m \tau) f(k)$. The linear process (11) is commonly adopted in the literature.

\section{Equilibrium}

At the equilibrium all markets clear (good, capital and labor). Observing that $k=K / L=h$, we obtain a dynamic system of equilibrium equations representing the interaction between the economic, environmental and biological spheres.

Proposition 3 Equilibrium dynamics are driven by a four-dimensional system:

$$
\begin{aligned}
\dot{\mu} & =f_{1}(\mu, k, N, P)=\mu[\theta+\delta-(1-\tau) \rho(k)] \\
\dot{k} & =f_{2}(\mu, k, N, P)=[(1-\tau) \rho(k)-\delta] k+(1-\tau) \omega(k)-c(\mu, N) \\
\dot{N} & =f_{3}(\mu, k, N, P)=N\left[\frac{N}{A(P)}-1\right]\left[1-\frac{N}{C(P)}\right] \\
\dot{P} & =f_{4}(\mu, k, N, P)=-a P+(b-m \tau) f(k)
\end{aligned}
$$

In this system, $\mu$ is a jump variable while $k, N$ and $P$ are predeterminate. Equation (12) and (13) represent the underlying Ramsey model, while equations (14) and (15) capture respectively the evolutions of biodiversity and pollution. With respect to the existing literature, pollution is view as a stock rather than a flow and both the carrying capacity and the Allee effect are introduced in terms of biodiversity. ${ }^{3}$

\section{Steady state}

Stationarity requires: $\dot{\mu}=\dot{k}=\dot{N}=\dot{P}=0$. Equation (12) gives the capital intensity at the steady state:

$$
k^{*}=\rho^{-1}\left(\frac{\theta+\delta}{1-\tau}\right)>0
$$

Replacing (16) into (15) gives the pollution level at the steady state:

$$
P^{*}=\frac{b-m \tau}{a} f\left(k^{*}\right)
$$

Assumption $4 \tau \leq b / m$.

Assumption 4 ensures that $P^{*} \geq 0$.

\footnotetext{
${ }^{3}$ See, among others, Ayong Le Kama (2001), Wirl (2004) and Bosi and Desmarchelier (2018b).
} 
Considering (14), the three possible biodiversity levels at the steady state are given by:

$$
N_{0}=0, N_{1}=A\left(P^{*}\right) \text { and } N_{2}=C\left(P^{*}\right)
$$

From equation (16), we obtain the consumption of steady state:

$$
c^{*}=\theta k^{*}+(1-\tau) \omega\left(k^{*}\right)
$$

Finally, for a given level of $N,(4)$ gives the shadow price of consumption:

$$
\mu^{*}=u_{c}\left(c^{*}, N^{*}\right)
$$

where $N^{*}$ is equal to $N_{0}, N_{1}$ or $N_{2}$.

Summing up, we get the following proposition.

Proposition 4 Let Assumptions 1 to 4 hold.

(1) If $A\left(P^{*}\right) \neq C\left(P^{*}\right)$, there are three steady states: $\left(\mu^{*}, k^{*}, P^{*}, N_{i}\right)$ with $i=0,1,2$.

(2) If $A\left(P^{*}\right)=C\left(P^{*}\right)$, there are two steady states: $\left(\mu^{*}, k^{*}, P^{*}, N_{0}\right)$ and $\left(\mu^{*}, k^{*}, P^{*}, N_{1}\right)=\left(\mu^{*}, k^{*}, P^{*}, N_{2}\right)$.

We expect the occurrence of a transcritical bifurcation when $A\left(P^{*}\right)=$ $C\left(P^{*}\right)$ (see below section 6 ).

In the following, for notational simplicity, if there is no ambiguity, we omit the asterisk to denote the steady state values, and we leave aside the steady state $N_{0}$ with wildlife annihilation and consequent mankind disappearance to focus on regimes with human survival.

The government can introduce and tune the green tax to mitigate the global warming and promote biodiversity. However, since Sinn (2008), we know that a higher green-tax rate can exacerbate instead of mitigate the global warming. Such a counter-intuitive effect, known as Green Paradox (GP) since the Sinn's pioneering work, can arise in many contexts. According Jensen et al. (2015), the GP can occur (1) when agents behave strategically in the resource markets (Gerlagh and Liski, 2011), (2) when resource and capital markets interact (Van der Meijden et al., 2015) or (3) when future policies are uncertain (Hoel, 2010). Recently, Bosi and Desmarchelier (2017a) have introduced a slightly different notion of $\mathrm{GP}^{4}$ and shown that it can arise in a Ramsey model when pollution comes from consumption, while Bosi and Desmarchelier (2018a) have also pointed out that this kind of GP can occur when the economy lies on the decreasing branch of an Environmental Kuznets Curve (EKC). ${ }^{5}$

To highlight the impossibility of a long-run GP in our context, we derive the impact of a green-tax rate (constant over time) on the pollution level of steady state.

\footnotetext{
${ }^{4}$ More focused on the long run than the short run and based on the comparative statics instead of the dynamics.

${ }^{5}$ The EKC is an inverted-U-shaped relation between income and pollution.
} 
Proposition 5 The impact of $\tau$ on the steady state is given by the following elasticities:

$$
\begin{aligned}
\frac{\tau}{k} \frac{\partial k}{\partial \tau} & =-\frac{\tau}{1-\tau} \frac{\sigma}{1-\alpha}<0 \\
\frac{\tau}{P} \frac{d P}{d \tau} & =\alpha \frac{\tau}{k} \frac{\partial k}{\partial \tau}-\frac{m \tau}{b-m \tau}<0 \\
\frac{\tau}{N_{1}} \frac{\partial N_{1}}{\partial \tau} & =\varepsilon_{A} \frac{\tau}{P} \frac{\partial P}{\partial \tau}<0 \\
\frac{\tau}{N_{2}} \frac{\partial N_{2}}{\partial \tau} & =\varepsilon_{C} \frac{\tau}{P} \frac{\partial P}{\partial \tau}>0 \\
\frac{\tau}{c} \frac{\partial c}{\partial \tau} & =\frac{1}{\gamma}\left(\left[\theta+(\theta+\delta) \frac{1-\alpha}{\sigma}\right] \frac{\tau}{k} \frac{\partial k}{\partial \tau}-\tau \frac{1-\alpha}{\alpha} \frac{\theta+\delta}{1-\tau}\right)<0
\end{aligned}
$$

where $\alpha=\alpha(k), \sigma=\sigma(k), \gamma=\gamma(k)$ and

$$
\gamma(k) \equiv \frac{\theta+\delta[1-\alpha(k)]}{\alpha(k)}=\frac{c}{k}
$$

Proposition 5 shows that a higher green tax always lowers the pollution level. Thereby, our context rules out the static GP introduced by Bosi and Desmarchelier (2017a). However, if the ultimate goal of a higher green tax is to promote the natural resource, another kind of GP arises around $N_{1}$ (let us call it the "Biodiversity Paradox" (BP)). Indeed, Proposition 5 points out that a higher green-tax rate lowers the biodiversity level $N_{1}$ in any case. Conversely, the green tax always improves the biodiversity level $N_{2}$ (here, no room for the $\mathrm{BP})$.

On the one side, how pollution shapes the Allee effect $\left(\varepsilon_{A}\right)$ creates the BP around $N_{1}$, while, on the other side, the impact of pollution on the carrying capacity $\left(\varepsilon_{C}\right)$ rules out the $\mathrm{BP}$ around $N_{2}$.

The effects of the tax rate on the other variables are trivial. Since the tax is levied on production, a higher rate gives an incentive to reduce the activities, lowering in turn the stock capital and, at the end, the consumption possibilities.

\section{$5 \quad$ Welfare analysis}

We have seen the opposite effects of a green tax on biodiversity around $N_{1}$ and $N_{2}$. Even an environment-friendly government mainly interested in sheltering or increasing the biodiversity, has to take care of the impact of consumption on social welfare. In this section, we try to understand whether a biodiversityoriented policy is really compatible with a welfare target.

Because of the representative household, welfare and utility maximizations are equivalent. We evaluate the social welfare at the steady state:

$$
W_{i}(\tau) \equiv \int_{0}^{\infty} e^{-\theta t} u\left(c(\tau), N_{i}(\tau)\right) d t=\frac{u\left(c(\tau), N_{i}(\tau)\right)}{\theta}
$$


with $i=0,1,2$.

We introduce the first-order elasticities of preferences to capture both the effect of consumption and biodiversity on the household's utility:

$$
\varepsilon_{c} \equiv \frac{c u_{c}}{u} \text { and } \varepsilon_{N} \equiv \frac{N u_{N}}{u}
$$

Differentiating (20), we compute the taxation impact on welfare as a new elasticity: ${ }^{6}$

$$
\frac{\tau W_{i}^{\prime}(\tau)}{W_{i}(\tau)}=\varepsilon_{c} \frac{\tau c^{\prime}(\tau)}{c(\tau)}+\varepsilon_{N} \frac{\tau N_{i}^{\prime}(\tau)}{N_{i}(\tau)}
$$

Proposition 6 Let Assumptions 1 to 4 hold. At the steady state given by $N_{1}$, a higher green-tax rate always reduces the welfare (biodiversity paradox), while, at the steady state given by $N_{2}$, a higher rate improves the welfare if and only if:

$$
0<\frac{\varepsilon_{c}}{\varepsilon_{N}}<-\frac{\frac{\tau N_{2}^{\prime}(\tau)}{N_{2}(\tau)}}{\frac{\tau c^{\prime}(\tau)}{c(\tau)}}
$$

The interpretation of the $\mathrm{BP}$ in Proposition 6 is straightforward. Indeed, around the steady state given by $N_{1}$, according to Proposition 5, a higher tax rate always lowers both the consumption and the biodiversity level, entailing in turn a drop in the welfare level. Focus now on $N_{2}$. According to Proposition 5 , a higher tax rate always increases biodiversity and reduces the consumption demand. The first effect (biodiversity) raises the welfare while the second one (consumption) lowers it. Hence, the welfare effect of the green tax is ambiguous. Interestingly, $\varepsilon_{c} / \varepsilon_{N}$ captures the slope of the indifference curve: condition (22) is satisfied when the slope of the indifference curve is sufficiently flat. This occurs when the representative household overvalues biodiversity with respect to consumption. In such a case, a higher green-tax rate allows the government to reach two objectives simultaneously: to increase both the biodiversity and the welfare level.

\section{Local dynamics}

Reconsidering Proposition (4) jointly with Assumption 3, we see that $N_{1}$ and $N_{2}$ move in opposite directions when pollution increases. In other words, the Allee threshold and the Carrying capacity approach one another as the pollution level rises. More precisely, according to (17), given $k$ of steady state, the larger the pollution inertia (the lower a), the higher the pollution level. Thus, we expect the existence of a critical value of pollution inertia such that $N_{1}$ and $N_{2}$ collide. Formally, such configuration corresponds to a transcritical bifurcation and represents a situation where the carrying capacity meets the Allee effect. In

\footnotetext{
${ }^{6}$ Notice that we do not exclude a negative value for $u, \varepsilon_{c}, \varepsilon_{N}$ or $W_{i}(\tau)$. Consider for instance the isoelastic specification (26) in section 7 with $\varepsilon>1$.
} 
this case, from a biological point of view, a mass extinction takes place: Nature can not support more individuals than the critical number needed to regenerate the biodiversity. Now, let us prove this intuitive conjecture.

To analyze the local dynamics, we linearize the dynamical system (12)-(15) around each non-trivial steady state $\left(N_{1}\right.$ or $\left.N_{2}\right)$.

Let $T, D, S_{2}$ and $S_{3}$ be respectively the trace, the determinant, the sums of minors of order two and three of the Jacobian matrix (given in the proof of the following lemma in the Appendix).

Lemma 7 The sum of minors of the Jacobian matrix of system (12)-(15) evaluated around the steady states with positive biodiversity level $N_{1}$ and $N_{2}$, is given by

$$
\begin{aligned}
T & =\theta+\varphi-a \\
S_{2} & =\theta(\varphi-a)-a \varphi+(1-\alpha)(\theta+\delta) \frac{\gamma}{\varepsilon_{c c}} \\
S_{3} & =(\varphi-a)(1-\alpha)(\theta+\delta) \frac{\gamma}{\varepsilon_{c c}}-a \theta \varphi+\gamma \psi(\theta+\delta) \frac{k}{P} \frac{b-m \tau}{1-\tau} \frac{\varepsilon_{c N}}{\varepsilon_{c c}} \\
D & =-a \varphi(1-\alpha)(\theta+\delta) \frac{\gamma}{\varepsilon_{c c}}
\end{aligned}
$$

where

$$
\begin{aligned}
\varphi & \equiv \frac{N}{A(P)}\left[1-\frac{N}{C(P)}\right]-\frac{N}{C(P)}\left[\frac{N}{A(P)}-1\right] \\
\psi & \equiv \frac{N \varepsilon_{A}}{A(P)}\left[\frac{N}{C(P)}-1\right]+\frac{N \varepsilon_{C}}{C(P)}\left[\frac{N}{A(P)}-1\right]
\end{aligned}
$$

$\varphi$ determines the sign of $D$ and, interestingly, $N=N_{1}=N_{2}$ implies $D=0$ (transcritical bifurcation).

From Proposition 4, we know that the economy has three steady states. From an ecological point of view, $N=N_{2}=C(P)$ is the most desirable when the carrying capacity exceeds the Allee effect and Nature can sustain a higher biodiversity level than what is needed for regeneration. Conversely, if the carrying capacity is lower than the Allee effect, a mass extinction occurs. When the carrying capacity meets the Allee effect, we get a critical point: $C(P)=A(P)$. In the following, we will focus only on the first case: $C(P) \geq A(P)$.

Notice that

$$
(\theta+\delta) \frac{k}{P} \frac{b-m \tau}{1-\tau}=a \alpha
$$

we obtain

$$
\begin{aligned}
T & =\theta+\varphi-a \\
S_{2} & =\theta(\varphi-a)-a \varphi+(1-\alpha)(\theta+\delta) \frac{\gamma}{\varepsilon_{c c}} \\
S_{3} & =(\varphi-a)(1-\alpha)(\theta+\delta) \frac{\gamma}{\varepsilon_{c c}}+a \alpha \gamma \psi \frac{\varepsilon_{c N}}{\varepsilon_{c c}}-a \theta \varphi \\
D & =-a \varphi(1-\alpha)(\theta+\delta) \frac{\gamma}{\varepsilon_{c c}}
\end{aligned}
$$


Proposition 8 If the carrying capacity exceeds the Allee effect at the steady state $\mathrm{N}_{2}\left(C\left(P^{*}\right)>A\left(P^{*}\right)\right)$, then local indeterminacy is excluded around $N_{2}$.

Proposition 9 If the carrying capacity exceeds the Allee effect $\left(C\left(P^{*}\right)>A\left(P^{*}\right)\right)$ and $a<\theta$ (strong pollution inertia), there is no room for local indeterminacy around $N_{1}$.

Propositions 8 and 9 shows that an equilibrium trajectory converging to $N_{1}$ or $N_{2}$ is locally unique. This result rules out the possibility of self-fulfilling prophecies from a local perspective.

As seen before, the restriction $C\left(P^{*}\right)>A\left(P^{*}\right)$ makes sense. Indeed, $C\left(P^{*}\right)<A\left(P^{*}\right)$ means that the carrying capacity is less than the survival threshold of the species. Courchamp et al. (1999) implicitly suppose $C\left(P^{*}\right)>$ $A\left(P^{*}\right)$. The transcritical bifurcation requires $A\left(P^{*}\right)=C\left(P^{*}\right)$ and implies that $N_{1}$ becomes stable when $C\left(P^{*}\right)<A\left(P^{*}\right)$ : thus, the stability of $N_{1}$ corresponds to a biological contradiction such that $C<N<A$ implies $\dot{N}>0$ according to (8).

Let

$$
a^{*} \equiv(b-m \tau) f(k)\left(\frac{\bar{A}}{\bar{C}}\right)^{\frac{1}{\varepsilon_{A}-\varepsilon_{C}}} \geq 0
$$

where $k$ is the steady state value.

Proposition 10 Consider the isoelastic functional forms (9). A transcritical bifurcation generically occurs at $a=a^{*}$.

The existence of a transcritical bifurcation is not surprising. Indeed, an increase in the pollution inertia (a lower $a$ ) raises the pollution level of steady state (see (17)) while Assumption 3 implies an increase of the Allee effect $\left(N_{1}\right)$ jointly with a decrease in the carrying capacity $\left(N_{2}\right)$. Thus, when $a$ decreases, $N_{1}$ and $N_{2}$ approach one another. $a^{*}$ is precisely the critical value such that the carrying capacity meets the Allee effect. In this respect, $a^{*}$ represents the threshold below which a mass extinction occurs: when $a$ falls below $a^{*}$, then $A\left(P^{*}\right)>C\left(P^{*}\right)$ and then Nature experiences a level of biodiversity lower than what is needed for its regeneration.

Focus now the effect of the green tax on the mass extinction threshold:

$$
\frac{\tau}{a^{*}} \frac{\partial a^{*}}{\partial \tau}=\alpha \frac{\tau}{k} \frac{\partial k}{\partial \tau}-\frac{m \tau}{b-m \tau}<0
$$

In other words, a higher tax rate, lowering $a^{*}$, increases the critical value of pollution inertia at which a mass extinction occurs. Thus, a simple Pigouvian tax can contribute to shelter the economy from a catastrophe. In terms of comparative statics, when household overvalues the biodiversity with respect to consumption (condition (22)), a higher green-tax rate can have a triple benefit: (1) a reduction in the pollution level jointly with an increase in the biodiversity according to Proposition 5, (2) a welfare improvement according to Proposition 6 ) and (3) a less likely anthropogenic mass extinction according to (25). 
In continuous time, persistent cycles can appear around the steady state through a Hopf bifurcation. In our environmental context, the limit cycles arising through this codimension-one bifurcation can be reinterpreted as biodiversity fluctuations over time. ${ }^{7}$

Let

$$
\begin{aligned}
& \varepsilon_{c N}^{-} \equiv \frac{\varepsilon_{c c}}{2 a \alpha \gamma \psi}\left(T S_{2}-2 \Omega-T \sqrt{S_{2}^{2}-4 D}\right) \\
& \varepsilon_{c N}^{+} \equiv \frac{\varepsilon_{c c}}{2 a \alpha \gamma \psi}\left(T S_{2}-2 \Omega+T \sqrt{S_{2}^{2}-4 D}\right)
\end{aligned}
$$

with $\Omega \equiv(\varphi-a)(1-\alpha)(\theta+\delta) \gamma / \varepsilon_{c c}-a \theta \varphi$.

Proposition 11 In the case of the isoelastic functional forms (9) with $C\left(P^{*}\right)>$ $A\left(P^{*}\right)$ (a carrying capacity higher than the Allee effect), a limit cycle generically arises near $N_{2}$ through a Hopf bifurcation if and only if $\varepsilon_{c N}=\varepsilon_{c N}^{+}$.

To understand why a limit cycle can surround $N_{2}$, we need to know the sign of $\varepsilon_{c N}^{+}$. A numerical simulation provided in the next section shows that, under a plausible calibration, $\varepsilon_{c N}^{+}<0$. Hence, a larger substitutability between biodiversity and consumption in terms of household's preferences seems to promote the occurrence of limit cycles. We can interpret this as follows: assume that the economy is in the steady state $N_{2}$ at the beginning and consider a sudden rise in the pollution level. According to Assumption 3, the carrying capacity decreases and, the biodiversity as well (remind that $N_{2}=C(P)$ ). Since biodiversity and consumption are substitutable $\left(\varepsilon_{c N}^{+}<0\right)$, households compensate the drop in utility due to the poorer biodiversity with an increase in their consumption demand. Accordingly, their savings lower and the aggregate capital level in turn, entailing a contraction in production and pollution at the end. Thus, a decrease in pollution follows the initial increase giving rise to the emergence of endogenous fluctuations.

Proposition 12 Consider the isoelastic functional forms (9). Assume that $C\left(P^{*}\right)>A\left(P^{*}\right)$ (a carrying capacity higher than the Allee effect). Through the Hopf bifurcation, the 3D-saddle-path stable steady state $N_{2}$ becomes $1 D$ saddle-path stable.

Proposition 12 shows how the economy looses its stability through the Hopf bifurcation. Before and after the Hopf bifurcation, the equilibrium is saddlepath stable. However, since the dynamic system (12)-(15) involves three predetermined variables (precisely, $k, P$ and $N$ ), the equilibrium stability requires at least a 3D stable manifold.

For now, we have considered only codimension-one bifurcations (transcritical and Hopf bifurcations). What happens when both of them take place in a neighborhood of a critical pair of parameters (codimension two)? In continuous time,

\footnotetext{
${ }^{7}$ The codimension is the number of parameters involved in the bifurcation.
} 
only three codimension-two bifurcations can be detected studying the eigenvalues of the Jacobian matrix: the Bogdanov-Takens, the Gavrilov-Guckenheimer and the double-Hopf bifurcations. ${ }^{8}$ However, in our ecological context, all these classes of bifurcations are ruled out, as we are now going to show.

Proposition 13 Consider the isoelastic functional forms (9). There is no room for a Bogdanov-Takens bifurcation.

The impossibility of Bogdanov-Takens bifurcation is quite interesting. Bosi and Desmarchelier (2018b) have shown that a Bogdanov-Takens bifurcation can arise in a Ramsey model with pollution viewed as a flow and a renewable resource whose reproduction obeys a standard logistic equation. Representing pollution as a stock seems more pertinent for some class of damages we are concerned by, such as the global warming. Moreover, the joint assumption of the Allee effect and the carrying capacity makes the picture even more realistic. Thus, a more realistic representation seems to reduce the dynamic complexity typically associated to a Bogdanov-Takens bifurcation.

Proposition 14 There is no room for a Gavrilov-Guckenheimer bifurcation.

Both Bogdanov-Takens and Gavrilov-Guckenheimer bifurcations imply the simultaneous occurrence of transcritical and Hopf bifurcations. Unsurprisingly, in our paper, these bifurcations are impossible. To grasp this point, focus for simplicity on the case of the isoelastic utility function (26) presented in section 7. As you can see checking the proof of Proposition $10, \varphi=\psi=0$ when the transcritical bifurcation occurs, while, giving a look to Proposition 11, in the isoelastic case considered, $\varepsilon_{c N}^{+}$vanishes when $\varphi=\psi=0$. Therefore, the simultaneous occurrence of Hopf and transcritical bifurcations is excluded.

Finally, we consider a codimension-two bifurcation involving a four-dimensional center manifold with, again, a negative but interesting result.

Proposition 15 When $N=N_{2}$ with $C\left(P^{*}\right)>A\left(P^{*}\right)$ (carrying capacity higher than the Allee effect), a double-Hopf bifurcation is impossible.

\section{Simulations}

We have characterized analytically the dynamics around steady state $N_{2}$ with the larger biodiversity and shown, in particular, the possibility of both transcritical and Hopf bifurcations. To convince the reader, let us illustrate these complex phenomena through a computer simulation. For this purpose, we adopt isoelastic preferences:

$$
u(c, N)=\frac{\left(c N^{\eta}\right)^{1-\varepsilon}}{1-\varepsilon}
$$

jointly with isoelastic Allee threshold and carrying capacity (see (9)).

\footnotetext{
${ }^{8}$ See Bosi and Desmarchelier (2017b) among others.
} 
We obtain

$$
\left[\begin{array}{cc}
\varepsilon_{c c} & \varepsilon_{c N} \\
\varepsilon_{N c} & \varepsilon_{N N}
\end{array}\right]=\left[\begin{array}{cc}
-\varepsilon & \eta(1-\varepsilon) \\
1-\varepsilon & \eta(1-\varepsilon)-1
\end{array}\right]
$$

Using (4), we get the explicit consumption function

$$
c(\mu, N)=\mu^{-\frac{1}{\varepsilon}} N^{\eta \frac{1-\varepsilon}{\varepsilon}}
$$

For simplicity, we assume a Cobb-Douglas technology, that is $y=f(k)=$ $A k^{\alpha}$ with $A>0$ and $\alpha \in(0,1)$. This implies that $\sigma=1$.

Under these specification, the steady state becomes

$$
\begin{aligned}
k & =\left[\frac{\alpha A(1-\tau)}{\theta+\delta}\right]^{\frac{1}{1-\alpha}} \\
P & =\frac{b-m \tau}{a} A k^{\alpha} \\
\mu & =c^{-\varepsilon} N_{i}^{\eta(1-\varepsilon)} \\
c & =\theta k+(1-\tau)(1-\alpha) A k^{\alpha}
\end{aligned}
$$

with

$$
N_{0}=0, N_{1}=\bar{A} P^{\varepsilon_{A}} \text { and } N_{2}=\bar{C} P^{\varepsilon_{C}}
$$

According to Proposition 11 and the matrix (27), a Hopf bifurcation arises near $N_{2}$, provided that $C\left(P^{*}\right)>A\left(P^{*}\right)$, if and only if:

$$
\eta=\eta_{H} \equiv \frac{\varepsilon}{\varepsilon-1} \frac{T S_{2}-2 \Omega+T \sqrt{S_{2}^{2}-4 D}}{2 a \alpha \gamma \psi}
$$

We perform an equilibrium continuation using the MATCONT package for MATLAB to illustrate the occurrence of a transcritical and a Hopf bifurcations around $\mathrm{N}_{2}$.

We adopt the following calibration:

\begin{tabular}{||l||l||l||l||l||l||l||l||l||l||l||l||l||}
\hline \hline Parameter & $A$ & $\bar{A}$ & $\bar{C}$ & $\alpha$ & $\theta$ & $\delta$ & $\varepsilon$ & $\varepsilon_{A}$ & $\varepsilon_{C}$ & $\tau$ & $b$ & $m$ \\
\hline \hline Value & 1 & 1 & 1 & 0.33 & 0.01 & 0.025 & 2 & 1 & -1 & 0.01 & 0.001 & 0.001 \\
\hline \hline
\end{tabular}

According to calibration (28), the transcritical bifurcation point is $a^{*}=$ 0.0029747. To implement the continuation, we choose a value of $a$ close to $a^{*}$ with $C\left(P^{*}\right)>A\left(P^{*}\right)$, say $a=0.003$.

(28) with $a=0.003$ yields $\eta_{H}=29.215$. The continuation exercise requires a value of $\eta$ close to $\eta_{H}$, say $\eta=28.5$.

The equilibrium continuation is represented in Fig. 1 in the parametric space 
$\{(a, \eta)\}$.

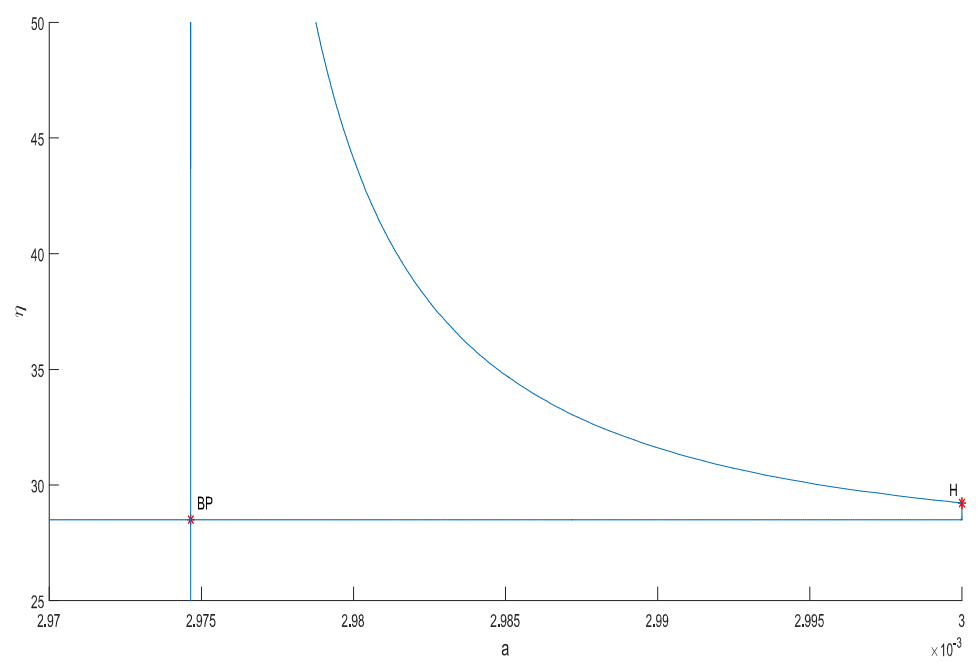

Fig.1. Equilibrium continuation

The curves $H$ and $B P$ are plotted by MATCONT point by point when, respectively, a Hopf and a transcritical bifurcation (Branch Point) are detected. Using the calibration $(28)$ jointly with $(a, \eta)=(0.003,28.5)$, we obtain the the following starting point for the continuation:

\begin{tabular}{||l||l||}
\hline \hline steady state & eigenvalues \\
\hline \hline$k=28.047$ & $\lambda_{1}=-0.039061$ \\
\hline \hline$P=0.99155$ & $\lambda_{2}=0.029359$ \\
\hline \hline$\mu=0.15191$ & $\lambda_{3}=-0.00020594-0.00652 i$ \\
\hline \hline$N_{2}=1.0085$ & $\lambda_{4}=-0.00020594+0.00652 i$ \\
\hline \hline
\end{tabular}

We remark that $C\left(P^{*}\right)=1.0085>A\left(P^{*}\right)=0.99155$.

The steady state we are focusing on, is 3D-saddle-path stable. Lowering $a$ and keeping $\eta=28.5$, MATCONT detects independently a transcritical bifurcation ( $B P$ in Fig. 1 ) when $a=0.0029746531 \approx a^{*}$. At this point, the steady state and the eigenvalues become:

\begin{tabular}{||l||l||}
\hline \hline steady state & eigenvalues \\
\hline \hline$k=28.047$ & $\lambda_{1}=-0.026232$ \\
\hline \hline$P=1$ & $\lambda_{2}=-0.00297465$ \\
\hline \hline$\mu=0.19347099$ & $\lambda_{3}=0$ \\
\hline \hline$N_{2}=1$ & $\lambda_{4}=0.036232$ \\
\hline \hline
\end{tabular}


We remark that, at this point, $C\left(P^{*}\right)=A\left(P^{*}\right)$ while, when $a<a^{*}$, $C\left(P^{*}\right)<A\left(P^{*}\right)$.

Now, we keep $a=0.003$ and we increase $\eta$. Independently, MATCONT detect a Hopf bifurcation $(H)$ when $\eta=29.215163 \approx \eta_{H}$. At this point, the steady state and the eigenvalues are given by:

\begin{tabular}{||l||l||}
\hline \hline steady state & eigenvalues \\
\hline \hline$k=28.047$ & $\lambda_{1}=-0.0392418$ \\
\hline \hline$P=0.99155031$ & $\lambda_{2}=0.0291258$ \\
\hline \hline$\mu=0.15099091$ & $\lambda_{3}=-0.00653447 i$ \\
\hline \hline$N_{2}=1.0085217$ & $\lambda_{4}=0.00653447 i$ \\
\hline \hline
\end{tabular}

MATCONT also reports the corresponding first Lyapunov coefficient:

$$
l_{1}=-3.861665 * 10^{-4}<0
$$

Since $l_{1}<0$, the Hopf bifurcation is supercritical and the limit cycle arising around the steady state is stable (see Fig. 2).

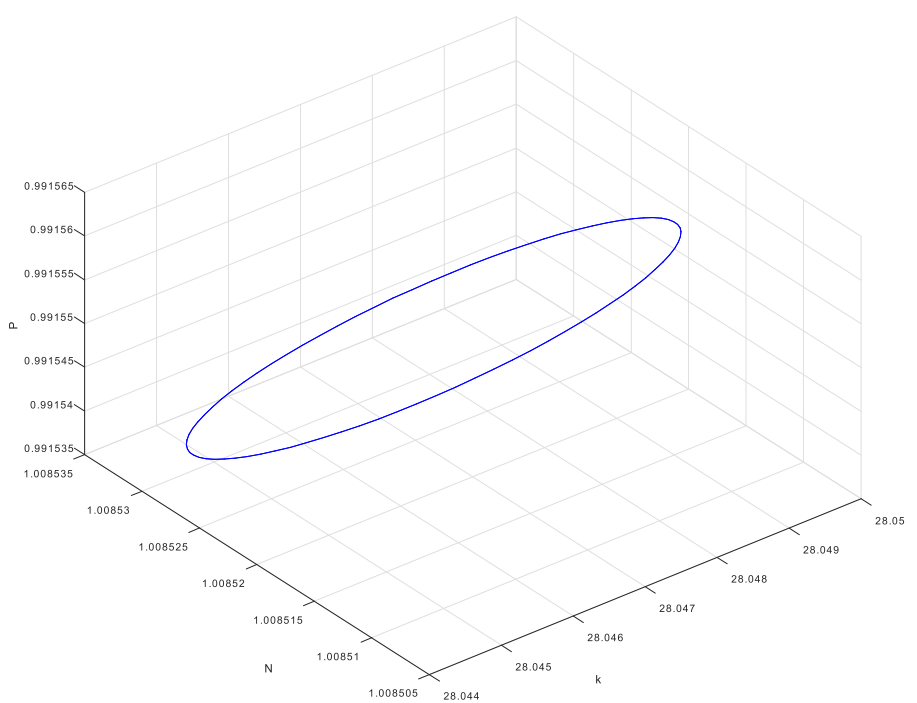

Fig.2. The stable limit cycle projected in the $(k, N, P)$-space

In Fig.1, the vertical line as well as the curve represent respectively both couples $(a, \eta)$ for which the transcritical and the Hopf bifurcations occur. That is, for all $(a, \eta)$ located on the left of the vertical line, the steady state is 2Dsaddle-path stable, for all $(a, \eta)$ located above the curve, the steady state is 
1D-saddle-path stable while the space between the vertical line and the curve is the parameter space for which the steady state is 3D-saddle-path stable.

To illustrate these stability changes and their sensitivity to the progressive values of $a$, let us fix $\eta=35$ and compute the eigenvalues at $N_{2}$ :

\begin{tabular}{||r||r||r||r||}
\hline \hline$a$ & 0.00297 & 0.00298 & 0.00299 \\
\hline \hline$\lambda_{1}$ & -0.023009 & -0.029510 & -0.035163 \\
\hline \hline$\lambda_{2}$ & 0.038029 & 0.034212 & 0.030553 \\
\hline \hline$\lambda_{3}$ & -0.006432 & $-0.0006401-0.003112 i$ & $0.000638-0.005192 i$ \\
\hline \hline$\lambda_{4}$ & 0.001568 & $-0.0006401+0.003112 i$ & $0.000638+0.005192 i$ \\
\hline \hline
\end{tabular}

\section{Conclusion}

We have considered a competitive Ramsey model with a pollution externality (viewed as a stock variable) coming from production and affecting the evolution of biodiversity (viewed as a renewable resource) through both the carrying capacity of Nature and the Allee effect. Biodiversity influences in turn the economy changing the marginal utility of consumption. In the long run, we have shown that the economy has three steady states: one exhibits zero biodiversity while the two others are driven by the Allee effect and the carrying capacity respectively. In the short run, we have proven the possible occurrence of limit cycles through a Hopf bifurcation around the steady state governed by the carrying capacity. Moreover, we have also pointed out the possibility of a transcritical bifurcation. This bifurcation takes place under a sufficiently high pollution inertia when the carrying capacity meets the Allee effect. In this sense, we can reinterpret the transcritical bifurcation point as a mass extinction threshold: below this tipping point the carrying capacity lowers the Allee effect. In this case, Nature supports only a level of biodiversity lower than what is needed for regeneration and Nature is dragged in an extinction vortex. By considering the impact of an environmental-friendly fiscal policy in the short and long run, we have shown that a higher green-tax rate can have a triple benefit: (1) a decrease in the pollution level jointly with an increase in biodiversity, (2) a welfare improvement and (3) a less likely anthropogenic mass extinction.

\section{Appendix}

\section{Proof of Proposition 2}

We apply the Pontryagin's maximum principle. The agent maximizes the intertemporal utility functional under the budget constraint (2). Setting the Hamiltonian $H=e^{-\theta t} u(c, N)+\lambda[(r-\delta) h+w-c]$, deriving the first-order conditions $\partial H / \partial c=0, \partial H / \partial h=-\dot{\lambda}$ and $\partial H / \partial \lambda=\dot{h}$, and defining $\mu \equiv \lambda e^{\theta t}$, we get (4), (5) and (6). Notice that the second-order Arrow-Mangasarian conditions are satisfied because Nature enters the utility function as an externality.

Proof of Proposition 3 
Consider (4), (5), (6) jointly with (1), (8) and (11).

Proof of Proposition 5

Simply differentiate (16), (17), (18) and (19).

Proof of Proposition 6

Apply Proposition 5 to expression (21). We have $W_{i}^{\prime}(\tau)>0$ if and only if

$$
0<\frac{\varepsilon_{c}}{\varepsilon_{N}}<-\frac{\frac{\tau N_{i}^{\prime}(\tau)}{N_{i}(\tau)}}{\frac{\tau c^{\prime}(\tau)}{c(\tau)}}
$$

If $i=1$, then the RHS is negative: the right inequality in (29) is violated and, therefore, $W_{1}^{\prime}(\tau)<0$. If $i=2$, then the RHS is positive: the right inequality in (29) is satisfied if and only if (22) holds.

Proof of Lemma 7

The Jacobian matrix is given by

$$
J \equiv\left[\begin{array}{llll}
\frac{\partial f_{1}}{\partial \mu} & \frac{\partial f_{1}}{\partial k} & \frac{\partial f_{1}}{\partial N} & \frac{\partial f_{1}}{\partial P} \\
\frac{\partial f_{2}}{\partial \mu} & \frac{\partial f_{2}}{\partial k} & \frac{\partial f_{2}}{\partial N} & \frac{\partial f_{2}}{\partial P} \\
\frac{\partial f_{3}}{\partial \mu} & \frac{\partial f_{3}}{\partial k} & \frac{\partial f_{3}}{\partial N} & \frac{\partial f_{3}}{\partial P} \\
\frac{\partial f_{4}}{\partial \mu} & \frac{\partial f_{4}}{\partial k} & \frac{\partial f_{4}}{\partial N} & \frac{\partial f_{4}}{\partial P}
\end{array}\right]=\left[\begin{array}{cccc}
0 & (\theta+\delta) \frac{1-\alpha(k)}{\sigma(k)} \frac{\mu}{k} & 0 & 0 \\
-c_{\mu} & \theta & -c_{N} & 0 \\
0 & 0 & \varphi & \psi \frac{N}{P} \\
0 & (b-m \tau) \frac{\theta+\delta}{1-\tau} & 0 & -a
\end{array}\right]
$$

where $\alpha=\alpha(k), \sigma=\sigma(k)$ and

$$
\varphi \equiv\left[\frac{N}{A(P)}-1\right]\left[1-\frac{N}{C(P)}\right]+\frac{N}{A(P)}\left[1-\frac{N}{C(P)}\right]-\frac{N}{C(P)}\left[\frac{N}{A(P)}-1\right]
$$

or, equivalently, (23), while $\psi$ is given by (24) because, around $N_{1}$ or $N_{2}$, the steady states we are interested in,

$$
\left[\frac{N}{A(P)}-1\right]\left[1-\frac{N}{C(P)}\right]=0
$$

We observe that

$$
\begin{aligned}
\varphi_{1} & \equiv \varphi\left(N_{1}\right)=1-\frac{A(P)}{C(P)} \\
\varphi_{2} & \equiv \varphi\left(N_{2}\right)=1-\frac{C(P)}{A(P)} \\
\psi_{1} & \equiv \psi\left(N_{1}\right)=\varepsilon_{A}\left[\frac{A(P)}{C(P)}-1\right] \\
\psi_{2} & \equiv \psi\left(N_{2}\right)=\varepsilon_{C}\left[\frac{C(P)}{A(P)}-1\right]
\end{aligned}
$$

because $N_{1}=A\left(P^{*}\right)$ and $N_{2}=C\left(P^{*}\right)$, and that, according to (7),

$$
\begin{aligned}
c_{\mu} & =\frac{1}{\varepsilon_{c c}} \frac{c}{\mu}=\frac{c}{k} \frac{1}{\varepsilon_{c c}} \frac{k}{\mu}=\frac{\pi}{\varepsilon_{c c}} \frac{k}{\mu} \\
c_{N} & =-\frac{\varepsilon_{c N}}{\varepsilon_{c c}} \frac{c}{N}=-\frac{c}{k} \frac{\varepsilon_{c N}}{\varepsilon_{c c}} \frac{k}{N}=-\pi \frac{\varepsilon_{c N}}{\varepsilon_{c c}} \frac{k}{N}
\end{aligned}
$$


Thus, we obtain

$$
J \equiv\left[\begin{array}{llll}
\frac{\partial f_{1}}{\partial \mu} & \frac{\partial f_{1}}{\partial k} & \frac{\partial f_{1}}{\partial N} & \frac{\partial f_{1}}{\partial P} \\
\frac{\partial f_{2}}{\partial \mu} & \frac{\partial f_{2}}{\partial k} & \frac{\partial f_{2}}{\partial N} & \frac{\partial f_{2}}{\partial P} \\
\frac{\partial f_{3}}{\partial \mu} & \frac{\partial f_{3}}{\partial k} & \frac{\partial f_{3}}{\partial N} & \frac{\partial f_{3}}{\partial P} \\
\frac{\partial f_{4}}{\partial \mu} & \frac{\partial f_{4}}{\partial k} & \frac{\partial f_{4}}{\partial N} & \frac{\partial f_{4}}{\partial P}
\end{array}\right]=\left[\begin{array}{cccc}
0 & (\theta+\delta) \frac{1-\alpha}{\sigma} \frac{\mu}{k} & 0 & 0 \\
-\frac{\gamma}{\varepsilon_{c c}} \frac{k}{\mu} & \theta & \gamma \frac{\varepsilon_{c N}}{\varepsilon_{c c}} \frac{k}{N} & 0 \\
0 & 0 & \varphi & \psi \frac{N}{P} \\
0 & (b-m \tau) \frac{\theta+\delta}{1-\tau} & 0 & -a
\end{array}\right]
$$

\section{Proof of Proposition 8}

We observe that the system (12)-(15) has one jump variable $(\mu)$ and three predetermined variables $(k, N$ and $P)$. In this case, local indeterminacy arises if and only if the four eigenvalues of $J$ are stable implying $D>0$ as a necessary (but not sufficient) condition for local indeterminacy. However, $C\left(P^{*}\right)>A\left(P^{*}\right)$ entails $\varphi_{2}<0$, that is $D<0$.

\section{Proof of Proposition 9}

If $C\left(P^{*}\right)>A\left(P^{*}\right)$, then $\varphi_{1}>0$ and $D>0$. Three cases are possible: (1) all eigenvalues are stable (local indeterminacy), (2) all eigenvalues are unstable or (3) two eigenvalues are stable while the other two are unstable. $a<\theta$ implies $T>0$ and rules out the first case (local indeterminacy).

Proof of Proposition 10

Consider functions (9). The two steady state coalesce when $N=N_{1}=N_{2}$, that is when $a=a^{*}$, holding if and only if $\varphi=\varphi_{1}=\varphi_{2}=D=0$.

Proof of Proposition 11

According to Bosi and Desmarchelier (2017b), a Hopf bifurcation generically arises in a $4 \mathrm{D}$-system if and only if $S_{2}=S_{3} / T+D T / S_{3}$ with $S_{3} / T>0$.

$S_{2}=S_{3} / T+D T / S_{3}$ if and only if

$$
\frac{S_{3}}{T}=\frac{S_{2} \pm \sqrt{S_{2}^{2}-4 D}}{2}
$$

that is if and only if $\varepsilon_{c N}=\varepsilon_{c N}^{ \pm}$. Moreover,

$$
\begin{aligned}
& \frac{S_{3}\left(\varepsilon_{c N}^{-}\right)}{T}=\frac{1}{2}\left[S_{2}-\sqrt{S_{2}^{2}-4 D}\right] \\
& \frac{S_{3}\left(\varepsilon_{c N}^{+}\right)}{T}=\frac{1}{2}\left[S_{2}+\sqrt{S_{2}^{2}-4 D}\right]
\end{aligned}
$$

Since $N=N_{2}$ with $C\left(P^{*}\right)>A\left(P^{*}\right)$, we get $D<0$. Then, $S_{3}\left(\varepsilon_{c N}^{-}\right) / T<$ $0<S_{3}\left(\varepsilon_{c N}^{+}\right) / T$. Therefore, only $\varepsilon_{c N}^{+}$satisfies the inequality required for a Hopf bifurcation.

Proof of Proposition 12

Jointly consider Propositions 8 and 11. $D<0$ means that at least one eigenvalue is stable and at least one is unstable. The Hopf bifurcation concerns the change in stability of two conjugate eigenvalues together.

Proof of Proposition 13 
According to Bosi and Desmarchelier (2017b), a Bogdanov-Takens bifurcation generically occurs in a $4 \mathrm{D}$-system if and only if $D=S_{3}=0$. According to the proof of Proposition 10, D $=0$ if and only if $a=a^{*}$. In this case, $\varphi=\varphi_{1}=\varphi_{2}=\psi=\psi_{1}=\psi_{2}=0$. Then, $S_{3}=-a(1-\alpha)(\theta+\delta) \gamma / \varepsilon_{c c}>0$ ruling out any Bogdanov-Takens bifurcation.

\section{Proof of Proposition 14}

According to Bosi and Desmarchelier (2017b), a Gavrilov-Guckenheimer bifurcation occurs if and only if $D=0$ such that $S_{3}=T S_{2}$ with $S_{2}>0$. From the proof of Proposition 10, we know that $D=0$ if and only if $a=a^{*}$. In this case, $N_{1}=A\left(P^{*}\right)=N_{2}=C\left(P^{*}\right)$. This implies $\varphi=0$ and, in turn,

$$
S_{2}=-a \theta+(1-\alpha)(\theta+\delta) \frac{\gamma}{\varepsilon_{c c}}<0
$$

\section{Proof of Proposition 15}

According to Bosi and Desmarchelier (2017b), a necessary (but not sufficient) condition for a double-Hopf bifurcation is $D>0$. Since $N=N_{2}$ and $C\left(P^{*}\right)>$ $A\left(P^{*}\right)$, then, $D<0$.

\section{References}

[1] Allee W.C. (1931). Animal Aggregations: A study in General Sociology. University of Chicago Press, Chicago.

[2] Ayong Le Kama A. (2001). Sustainable growth, renewable resources and pollution. Journal of Economic Dynamics \& Control 25, 1911-1918.

[3] Barnosky A.D., N. Matzke, S. Tomiya, G. O. Wogan, B. Swartz., T.B. Quental, C. Marshall, J.L. McGuire, E.L. Lindsey, K.C. Maguire, B. Mersey and E.A. Ferrer (2011). Has the Earth's sixth mass extinction already arrived? Nature 471, 51-57.

[4] Beltratti A., G. Chichilnisky and G. Heal (1994). Sustainable growth and the Green Golden Rule. In: Goldin I., L.A. Winters (eds.), The Economics of Sustainable Development, Cambridge University Press, Cambridge.

[5] Bosi S. and D. Desmarchelier (2017a). Are the Laffer curve and the Green Paradox mutually exclusive? Journal of Public Economic Theory 19, 937956.

[6] Bosi S. and D. Desmarchelier (2017b). Local bifurcations of three and fourdimensional systems: a tractable characterization with economic applications. Working paper Ec-02/17, Department of Economics, European University at St. Petersburg.

[7] Bosi S. and D. Desmarchelier (2018a). Limit cycles under a negative effect of pollution on consumption demand: the role of an environmental Kuznets curve. Environmental and Resource Economics, 69, 343-363. 
[8] Bosi S. and D. Desmarchelier (2018b). Natural cycles and pollution. Forthcoming in Mathematical Social Sciences.

[9] Brook B.W., N.S. Sodhi and C.J.A. Bradshaw (2008). Synergies among extinction drivers under global change. Trends in Ecology and Evolution $23,453-460$.

[10] Ceballos G., P.R. Ehrlich., A.D. Barnosky, A. García, R.M. Pringle and T.M. Palmer (2015). Accelerated modern human-induced species losses: entering the sixth mass extinction. Science Advances 1, 1-5.

[11] Celli G. and B. Maccagnani (2003). Honey bees as bioindicators of environmental pollution. Bulletin of Insectology 56, 137-139.

[12] Courchamp F., T. Clutton-Brock and B. Grenfell (1999). Inverse density dependence and the Allee effect. Trends in Ecology and Evolution 14, 405410 .

[13] Gerlagh R. and M. Liski (2011). Strategic resource dependence. Journal of Economic Theory 146, 699-727.

[14] Hoel M. (2010). Climate change and carbon tax expectations. CESIFO Working Paper n. 2966.

[15] Jensen S., K. Mohlin., K. Pittel, and T. Sterner (2015). An introduction to the green paradox: the unintended consequences of climate policies. Review of Environmental Economics and Policy 9, 246-265.

[16] Memmott J., N.M. Waser and M.V. Price (2004). Tolerance of pollination networks to species extinctions. Proceeding of the Royal Society B 271, 2605-2611.

[17] Sinn H.-W. (2008). Public policies against global warming: a supply side approach. International Tax and Public Finance 15, 360-394.

[18] Van der Meijden G., F. Van der Ploeg and C. Withagen (2015). International capital markets, oil producers and the Green Paradox. European Economic Review 76, 275-297.

[19] Wirl F. (2004). Sustainable growth, renewable resources and pollution: thresholds and cycles. Journal of Economic Dynamics \& Control 28, 11491157. 